\title{
Microwave-assisted Synthesis of New 1,3-thiazolidin-4-ones and Evaluation of Their Anticancer Efficacy
}

\author{
Zhengyue Ma \\ Key Laboratory for Pharmaceutical Quality Control of Hebei Province \\ College of Pharmaceutical Science, Hebei University \\ Baoding, Hebei, 071002, China \\ E-mail: mazhengy@126.com \\ Xinghua Zhang \\ Key Laboratory for Pharmaceutical Quality Control of Hebei Province \\ College of Pharmaceutical Science, Hebei University \\ Baoding, Hebei, 071002, China \\ E-mail: zhangxinghua20061984@126.com
}

Ligai Bai, Yajun Zheng \& Gengliang Yang (Corresponding author)

Key Laboratory for Pharmaceutical Quality Control of Hebei Province

College of Pharmaceutical Science, Hebei University

Baoding, Hebei, 071002, China

Received: February 21, 2011

Accepted: March 11, 2011

doi:10.5539/mas.v5n3p207

We are grateful for financial support by the National Natural Science Foundation of China (Grant Nos. 20375010, and 20675084), Program for Science and Technology Development of Hebei Province (Grant Nos. $06276479 B$ and $07276407 D$ ).

\begin{abstract}
An efficient method for the synthesis of thiazolidinones from the microwave-assisted, three-component mixture of an aromatic aldehyde, a 2-amino- $4 H$-benzothiopyrano[4,3- $d]$ thiazole and meraptoacetic acid is described. The transformation of the amino group of 2-amino-4H-[1]benzothiopyrano[4,3- $d$ ] thiazo- les into a thiazolidinone ring has not been reported to date. In vitro their anticancer activity was evaluated by MTT method. The results of bioactivities evaluation showed the compound 4 exhibited the best anticancer activity and its $\mathrm{IC}_{50}$ was $5.76 \mu \mathrm{M}$, $6.19 \mu \mathrm{M}, 6.67 \mu \mathrm{M}$ against gastric cancer cells (SGC-7901), cervical cancer cells (Hela) and lung cancer cells (A-549), respectively.

Keywords: 1,3-thiazolidin-4-ones, $4 H$-thiochromeno[4,3- $d$ ]thiazol-2-amines, Anticancer activity, DCC, Microwave-assisted synthesis

\section{Introduction}

Thiazoles are one of the most intensively investigated classes of aromatic five membered hetrocycles. Thiazole derivatives find a variety of applications ranging from anti-fungal and anti-bacterial through to CNS regulators of commercially successful diuretics. All these facts were driving force to develop novel thiozole derivatives with wide structural varation.

Thiazolidin-4-ones have played an important role in medicinal chemistry. Moreover they have been studied extensively because of their ready accessibility, diverse chemical reactivity and brode spectrum of biological activity, such as anti-HIV (Ravindra, K.R., 2007; Murugesan, V., 2009; Balzarini, J.,2007; Küçükgüzel, G., 2006), anti-mycobacterial (Aridoss, G., 2009), anti-proliferative (Ottana, R., 2005), anti-microbial (Mujeebur, R.V.P., 2005; Vicini, P., 2008; Vicini, P., 2006), anti-inflammatory (Ottana, R., 2005), anti-cancer (Chen, Hua,
\end{abstract}


2008), anti-fungal (Dandia, A., 2006). Studies revealed that a 2-(thiazolyl)substituent at N-3 of a 1,3-thiazolidin-4-one displayed anti-microbial and anti-HIV activitiy (Ravindra, K.R., 2007). Thiochromanones also had been reported to possess important biological activities. In this paper, thiochromanones (1) as the starting material were transformed into $4 H$-thiochromeno[4,3- $d]$ thiazol-2-amines (2) and providing amino parts. The transformation of the amino group of 2-amino-4H-[1]benzothiopyrano[4,3- $d$ ] thiazoles into a thiazolidinone ring has not been reported to date. It was worthy to explore this unusual heterocyclic system and investigate any potential anticancer activity.

\section{Experimental}

\subsection{Chemistry}

MW experiments were carried out in Microwave synthesizer. Melting points of all compounds synthesized were determined with a SGW X-4 melting point apparatus. The ${ }^{1} \mathrm{H}$ NMR spectra were recorded in $\mathrm{CDCl}_{3}$ at $600 \mathrm{MHz}$ with a AVANCE 600 spectrometer (internal standard TMS, $\delta=0.0 \mathrm{ppm}$ ). IR spectra were recorded on a Bio-Rad FTS-40 spectrometer (KBr).The MS spectra were measured on LC-MSD Trap XCT spectrometer. Elemental analysis was performed on a Carlo Erba-1106 instrument and the results were in acceptable range. All materials were commercially available and used as received unless otherwise noted. Substituted $4 H$-thiochromeno-[4,3- $d]$ thiazol-2-amines were prepared according to method reported (Zhan Fuxun, 2009).

\subsection{Preparation of compounds 3}

Substituted $4 H$-thiochromeno[4,3- $d$ ] thiazol-2-amine $(5.00 \mathrm{mmol})$, aromatic aldhyde $(5.00 \mathrm{mmol})$ and mercaptoacetic acid $(10.00 \mathrm{mmol})$ were added in microwave reaction tube $(10 \mathrm{~mL})$, added toluene $(4 \mathrm{~mL})$ dissolved the mixture, then added DCC (Dicyclohexylcarbodiimide, $10.00 \mathrm{mmol}$ ). The reaction tube was irradiated at $140{ }^{\circ} \mathrm{C}$ for $10 \mathrm{~min}$. Upon completion, colled to room temperature, the solids were filtered and added $\mathrm{CHCl}_{3}(20 \mathrm{~mL})$ to the filtrate, sequentially washed with saturated $\mathrm{NaHCO}_{3}, \mathrm{H}_{2} \mathrm{O}$, brine and then dried over anhydrous $\mathrm{MgSO}_{4}$. Solvent was removed under reduced presser to provide a crude product which was purified by column chromatography (silica gel, ethyl acetate: petroleum=1:40-1:10(v/v)) to afford pure 3 .

\subsubsection{3-(8-Chloro-4H-thiochromeno[4,3-d]thiazol-2-yl)-2-(4-methoxyphenyl)thiazolidin-4-one (3a)}

Yield $50 \%$ of red brown solid, m.p. $123-125^{\circ} \mathrm{C}$, UV-vis $(\mathrm{MeOH}) \lambda_{\max }: 238 \mathrm{~nm}$; IR (KBr) $v: 3010,1689,1610$, $1600,1089,1471,877,837 \mathrm{~cm}^{-1} ;{ }^{1} \mathrm{H} \mathrm{NMR}\left(\mathrm{CDCl}_{3}, 600 \mathrm{MHz}\right) \delta: 3.78\left(\mathrm{~s}, 3 \mathrm{H}, \mathrm{OCH}_{3}\right), 3.87(\mathrm{~d}, J=16.43 \mathrm{~Hz}, 1 \mathrm{H}$, $\left.\mathrm{COCH}_{2} \mathrm{~S}\right), 4.03\left(\mathrm{~d}, J=15.08 \mathrm{~Hz}, 1 \mathrm{H}, \mathrm{COCH}_{2} \mathrm{~S}\right), 4.08-4.17\left(\mathrm{~m}, 2 \mathrm{H}, \mathrm{SCH}_{2}\right), 6.63(\mathrm{~s}, 1 \mathrm{H}, \mathrm{NCHS}), 6.88(\mathrm{~d}, J=$ $8.74 \mathrm{~Hz}, 2 \mathrm{H}, \mathrm{Ar}-\mathrm{H}), 7.08$ (dd, $J=8.31,2.33 \mathrm{~Hz}, 1 \mathrm{H}, \mathrm{Ar}-\mathrm{H}), 7.16$ (d, $J=8.30 \mathrm{~Hz}, 1 \mathrm{H}, \mathrm{Ar}-\mathrm{H}), 7.34$ (d, $J=8.75$ $\mathrm{Hz}, 2 \mathrm{H}, \mathrm{Ar}-\mathrm{H}), 7.60(\mathrm{~d}, J=2.31 \mathrm{~Hz}, 1 \mathrm{H}, \mathrm{Ar}-\mathrm{H})$; MS (APCI) $m / z$ : $446.8(\mathrm{M}+\mathrm{H})^{+}, 448.8(\mathrm{M}+2+\mathrm{H})^{+}$. Anal. Calcd for $\mathrm{C}_{20} \mathrm{H}_{15} \mathrm{ClN}_{2} \mathrm{O}_{2} \mathrm{~S}_{3}$ : C 53.74, H 3.38, N 6.88, S 21.57; Found C 53.69, H 3.33, N 6.83, S 21.53.

\subsubsection{3-(8-Chloro-4H-thiochromeno[4,3-d]thiazol-2-yl)-2-(furan-2-yl)thiazolidin-4-one (3b)}

Yield $60 \%$ of pale brown solid, m.p. $177-179{ }^{\circ} \mathrm{C}$, UV-vis $(\mathrm{MeOH}) \lambda_{\max }: 238 \mathrm{~nm}$; IR (KBr) $v: 1689,1620,1588$, 1506, 1488, $1458 \mathrm{~cm}^{-1}$; ${ }^{1} \mathrm{H}$ NMR $\left(\mathrm{CDCl}_{3}, 600 \mathrm{MHz}\right) \delta: 3.77$ (d, $\left.J=16.06 \mathrm{~Hz}, 1 \mathrm{H}, \mathrm{COCH}_{2} \mathrm{~S}\right), 4.13-4.06(\mathrm{~m}, 2 \mathrm{H}$, $\left.\mathrm{SCH}_{2}\right), 4.28\left(\mathrm{~d}, J=16.13 \mathrm{~Hz}, 1 \mathrm{H}, \mathrm{COCH}_{2} \mathrm{~S}\right), 6.33(\mathrm{dd}, J=3.28,1.84 \mathrm{~Hz}, 1 \mathrm{H}$, furan-H), $6.43(\mathrm{~d}, J=3.25 \mathrm{~Hz}$, $1 \mathrm{H}$, furan-H), 6.74 (s, $1 \mathrm{H}, \mathrm{NCHS}), 7.11$ (dd, $J=8.32,2.39 \mathrm{~Hz}, 1 \mathrm{H}, \mathrm{Ar}-\mathrm{H}), 7.20$ (d, $J=8.31 \mathrm{~Hz}, 1 \mathrm{H}, \mathrm{Ar}-\mathrm{H}), 7.36$ $\left(\mathrm{d}, J=1.08 \mathrm{~Hz}, 1 \mathrm{H}\right.$, furan-H), $7.76(\mathrm{~d}, J=2.32 \mathrm{~Hz}, 1 \mathrm{H}, \mathrm{Ar}-\mathrm{H})$; MS (APCI) $m / z: 406.5(\mathrm{M}+\mathrm{H})^{+}, 408.5$ $(\mathrm{M}+2+\mathrm{H})^{+}$. Anal. Calcd for $\mathrm{C}_{17} \mathrm{H}_{11} \mathrm{ClN}_{2} \mathrm{O}_{2} \mathrm{~S}_{3}$ : C 50.18, H 2.72, N 6.88, S 23.64; Found C 50.14, H 2.76, N 6.83, S 23.67.

\subsubsection{3-(8-Chloro-4H-thiochromeno[4,3-d]thiazol-2-yl)-2-(4-nitrophenyl)thiazolidin-4-one (3c)}

Yield $88 \%$ of red solid, m.p. $198-199^{\circ} \mathrm{C}$, UV-vis $(\mathrm{MeOH}) \lambda_{\max }: 240 \mathrm{~nm}$; IR $(\mathrm{KBr}) v: 1685,1620,3078,1517$, $1458,1344,829 \mathrm{~cm}^{-1} ;{ }^{1} \mathrm{H}$ NMR $\left(\mathrm{CDCl}_{3}, 600 \mathrm{MHz}\right) \delta: 3.96\left(\mathrm{~d}, J=16.47 \mathrm{~Hz}, 1 \mathrm{H}, \mathrm{COCH}_{2} \mathrm{~S}\right), 4.09(\mathrm{~d}, J=15.16$ $\left.\mathrm{Hz}, 1 \mathrm{H}, \mathrm{COCH}_{2} \mathrm{~S}\right), 4.11-4.18\left(\mathrm{~m}, 2 \mathrm{H}, \mathrm{SCH}_{2}\right), 6.74$ (s, $\left.1 \mathrm{H}, \mathrm{NCHS}\right), 7.10$ (dd, $\left.J=8.32,2.37 \mathrm{~Hz}, 1 \mathrm{H}, \mathrm{Ar}-\mathrm{H}\right), 7.19$ $(\mathrm{d}, J=8.30 \mathrm{~Hz}, 1 \mathrm{H}, \mathrm{Ar}-\mathrm{H}), 7.46(\mathrm{~d}, J=2.31 \mathrm{~Hz}, 1 \mathrm{H}, \mathrm{Ar}-\mathrm{H}), 7.57(\mathrm{~d}, J=8.77 \mathrm{~Hz}, 2 \mathrm{H}, \mathrm{Ar}-\mathrm{H}), 8.27(\mathrm{~d}, J=8.77$ $\mathrm{Hz}, 2 \mathrm{H}, \mathrm{Ar}-\mathrm{H})$; MS (APCI) $m / z: 461.8(\mathrm{M}+\mathrm{H})^{+}, 463.8(\mathrm{M}+2+\mathrm{H})^{+}$. Anal. Calcd for $\mathrm{C}_{19} \mathrm{H}_{12} \mathrm{ClN}_{3} \mathrm{O}_{3} \mathrm{~S}_{3}: \mathrm{C} 49.40, \mathrm{H}$ 2.62, N 9.10, S 20.82; Found C 49.36, H 2.58, N 9.06, S 20.79.

\subsubsection{3-(8-Chloro-4H-thiochromeno[4,3-d]thiazol-2-yl)-2-phenylthiazolidin-4-one (3d)}

Yield $63 \%$ of yellow solid, m.p. $183-186{ }^{\circ} \mathrm{C}$, UV-vis (MeOH) $\lambda_{\max }$ : $238 \mathrm{~nm}$; IR (KBr) $v: 3029,1685,1621,1490$, $1456 \mathrm{~cm}^{-1}$; ${ }^{1} \mathrm{H}$ NMR $\left(\mathrm{CDCl}_{3}, 600 \mathrm{MHz}\right) \delta: 3.87\left(\mathrm{~d}, J=16.44 \mathrm{~Hz}, 1 \mathrm{H}, \mathrm{COCH}_{2} \mathrm{~S}\right), 4.03(\mathrm{~d}, J=15.10 \mathrm{~Hz}, 1 \mathrm{H}$, COCHS), 4.09-4.16 (m, 2H, SCH 2$), 6.67$ (d, $J=0.86 \mathrm{~Hz}, 1 \mathrm{H}, \mathrm{NCHS}), 7.07$ (dd, $J=8.31,2.37 \mathrm{~Hz}, 1 \mathrm{H}, \mathrm{Ar}-\mathrm{H})$, $7.15(\mathrm{~d}, J=8.30 \mathrm{~Hz}, 1 \mathrm{H}, \mathrm{Ar}-\mathrm{H}), 7.28-7.32(\mathrm{~m}, 1 \mathrm{H}, \mathrm{Ar}-\mathrm{H}), 7.35-7.39(\mathrm{~m}, 4 \mathrm{H}, \mathrm{Ar}-\mathrm{H})$; MS (APCI) $m / z: 416.8$ $(\mathrm{M}+\mathrm{H})^{+}, 418.8(\mathrm{M}+2+\mathrm{H})^{+}$. Anal. Calcd for $\mathrm{C}_{19} \mathrm{H}_{13} \mathrm{ClN}_{2} \mathrm{OS}_{3}$ : C 54.73, H 3.14, N 6.72, S 23.07; Found C 54.67, H 
3.09, N 6.69, S 23.11 .

2.2.5 3-(8-Chloro-4H-thiochromeno[4,3-d]thiazol-2-yl)-2-(4-(dimethylamino)phenyl)thiazolidin-4-one (3e)

Yield 55\% of pale yellow solid, m.p. $129-131{ }^{\circ} \mathrm{C}$, UV-vis (MeOH) $\lambda_{\max }: 240 \mathrm{~nm}$; IR (KBr) $v: 2967,2855,1685$, $1620,1588,1490,1460,1450,1385 \mathrm{~cm}^{-1} ;{ }^{1} \mathrm{H} \mathrm{NMR}\left(\mathrm{CDCl}_{3}, 600 \mathrm{MHz}\right) \delta: 3.08\left(\mathrm{~s}, 3 \mathrm{H}, \mathrm{CH}_{3}\right), 3.10\left(\mathrm{~s}, 3 \mathrm{H}, \mathrm{CH}_{3}\right)$, $3.86\left(\mathrm{~d}, J=16.44 \mathrm{~Hz}, 1 \mathrm{H}, \mathrm{COCH}_{2} \mathrm{~S}\right), 4.06\left(\mathrm{~d}, J=28.16 \mathrm{~Hz}, 1 \mathrm{H}, \mathrm{COCH}_{2} \mathrm{~S}\right), 4.15\left(\mathrm{~s}, 2 \mathrm{H}, \mathrm{SCH}_{2}\right), 6.62(\mathrm{~s}, 1 \mathrm{H}$, NCHS), 6.69 (d, $J=10.27 \mathrm{~Hz}, 1 \mathrm{H}, \mathrm{Ar}-\mathrm{H}), 6.73$ (d, $J=8.85 \mathrm{~Hz}, 1 \mathrm{H}, \mathrm{Ar}-\mathrm{H}), 7.10$ (dd, $J=20.48,2.26 \mathrm{~Hz}, 1 \mathrm{H}, \mathrm{Ar}-\mathrm{H}$ ), $7.18(\mathrm{~d}, J=24.94 \mathrm{~Hz}, 1 \mathrm{H}, \mathrm{Ar}-\mathrm{H}), 7.30(\mathrm{~d}, J=8.73 \mathrm{~Hz}, 1 \mathrm{H}, \mathrm{Ar}-\mathrm{H}), 7.74(\mathrm{~d}, J=8.75 \mathrm{~Hz}, 1 \mathrm{H}, \mathrm{Ar}-\mathrm{H}), 7.87$ (d, $J=8.47$ $\mathrm{Hz}, 1 \mathrm{H}, \mathrm{Ar}-\mathrm{H})$; MS (APCI) $m / z: 459.8(\mathrm{M}+\mathrm{H})^{+}, 461.8(\mathrm{M}+2+\mathrm{H})^{+}$. Anal. Calcd for $\mathrm{C}_{21} \mathrm{H}_{18} \mathrm{ClN}_{3} \mathrm{OS}_{3}: \mathrm{C} 54.83, \mathrm{H}$ 3.94, N 9.13, S 20.91; Found C 54.79, H 3.87, N 9.09, S 20.96.

2.2.6 3-(8-Chloro-4H-thiochromeno[4,3-d]thiazol-2-yl)-2-(4-chlorophenyl)thiazolidin-4-one (3f)

Yield $86 \%$ of brown solid, m.p. $147-151{ }^{\circ} \mathrm{C}$, UV-vis $(\mathrm{MeOH}) \lambda_{\max }: 238 \mathrm{~nm}$; IR (KBr) $v: 1697,1621,1521,1488$, $1458 \mathrm{~cm}^{-1} ;{ }^{1} \mathrm{H}$ NMR $\left(\mathrm{CDCl}_{3}, 600 \mathrm{MHz}\right) \delta: 3.77\left(\mathrm{~d}, J=16.03 \mathrm{~Hz}, 1 \mathrm{H}, \mathrm{COCH}_{2} \mathrm{~S}\right)$, , 4.04-4.10 (m, 2H, $\left.\mathrm{SCH}_{2}\right), 4.29$ $\left(\mathrm{d}, J=15.96 \mathrm{~Hz}, 1 \mathrm{H}, \mathrm{COCH}_{2} \mathrm{~S}\right), 6.32(\mathrm{~s}, 1 \mathrm{H}, \mathrm{NCHS}), 6.42(\mathrm{~d}, J=3.12 \mathrm{~Hz}, 1 \mathrm{H}, \mathrm{Ar}-\mathrm{H}), 6.73(\mathrm{~s}, 1 \mathrm{H}, \mathrm{Ar}-\mathrm{H}), 7.09$ $(\mathrm{d}, J=7.18 \mathrm{~Hz}, 1 \mathrm{H}, \mathrm{Ar}-\mathrm{H}), 7.25(\mathrm{~d}, J=9.11 \mathrm{~Hz}, 2 \mathrm{H}, \mathrm{Ar}-\mathrm{H}), 7.35(\mathrm{~s}, 1 \mathrm{H}, \mathrm{Ar}-\mathrm{H}), 7.44(\mathrm{~d}, J=10.19 \mathrm{~Hz}, 1 \mathrm{H}$, Ar-H); MS (APCI) $m / z: 450.8(\mathrm{M}+\mathrm{H})^{+}, 452.8(\mathrm{M}+2+\mathrm{H})^{+}$. Anal. Calcd for $\mathrm{C}_{19} \mathrm{H}_{12} \mathrm{Cl}_{2} \mathrm{~N}_{2} \mathrm{OS}$ : $\mathrm{C} 50.55, \mathrm{H} 2.68, \mathrm{~N}$ 6.21, S 21.31; Found C 50.49, H 2.62, N 6.26, S 21.28.

\subsubsection{2-(Benzo[d][1,3]dioxol-5-yl)-3-(8-chloro-4H-thiochromeno[4,3-d]thiazol-2-yl)-thiazoli-din-4-one (3g)}

Yield $48 \%$ of pale brown solid, m.p. $154-157^{\circ} \mathrm{C}$, UV-vis (MeOH) $\lambda_{\max }: 240 \mathrm{~nm}$; IR (KBr) $v: 3080,2923,2854$, $1683,1625,1588,1521,1488,1338,1245,1033 \mathrm{~cm}^{-1} ;{ }^{1} \mathrm{H}$ NMR $\left(\mathrm{CDCl}_{3}, 600 \mathrm{MHz}\right) \delta: 3.89(\mathrm{~d}, J=16.47 \mathrm{~Hz}, 1 \mathrm{H}$, $\left.\mathrm{COCH}_{2} \mathrm{~S}\right), 4.07\left(\mathrm{~d}, J=15.08 \mathrm{~Hz}, 1 \mathrm{H}, \mathrm{COCH}_{2} \mathrm{~S}\right), 4.19-4.12\left(\mathrm{~m}, 2 \mathrm{H}, \mathrm{SCH}_{2}\right), 5.97(\mathrm{dd}, J=9.16,1.22 \mathrm{~Hz}, 2 \mathrm{H}$, $\left.\mathrm{OCH}_{2} \mathrm{O}\right), 6.62(\mathrm{~s}, 1 \mathrm{H}, \mathrm{NCHS}), 6.80(\mathrm{~d}, J=8.05 \mathrm{~Hz}, 1 \mathrm{H}, \mathrm{Ar}-\mathrm{H}), 6.85(\mathrm{~d}, J=1.67 \mathrm{~Hz}, 1 \mathrm{H}, \mathrm{Ar}-\mathrm{H}), 6.93(\mathrm{dd}, J=$ 8.07, 1.67 Hz, 1H, Ar-H), 7.11 (dd, $J=8.31,2.36 \mathrm{~Hz}, 1 \mathrm{H}, \mathrm{Ar}-\mathrm{H}), 7.19$ (d, $J=8.30 \mathrm{~Hz}, 1 \mathrm{H}, \mathrm{Ar}-\mathrm{H}), 7.62(\mathrm{~d}, J=$ $2.29 \mathrm{~Hz}, 1 \mathrm{H}, \mathrm{Ar}-\mathrm{H})$; $\mathrm{MS}(\mathrm{APCI}) \mathrm{m} / z: 460.8(\mathrm{M}+\mathrm{H})^{+}, 462.8(\mathrm{M}+2+\mathrm{H})^{+}$. Anal. Calcd for $\mathrm{C}_{20} \mathrm{H}_{13} \mathrm{ClN}_{2} \mathrm{O}_{3} \mathrm{~S}_{3}: \mathrm{C} 52.11$, H 2.84, N 6.08, S 20.87; Found C 52.08, H 2.79, N 6.12, S 20.83.

2.2.8 2-(Furan-2-yl)-3-(8-methyl-4H-thiochromeno[4,3-d]thiazol-2-yl)thiazolidin-4-one (3h)

Yield $62 \%$ of brown solid, m.p. $163-165^{\circ} \mathrm{C}$, UV-vis $(\mathrm{MeOH}) \lambda_{\max }: 238 \mathrm{~nm}$; IR $(\mathrm{KBr}) v$ : 2923, 2852, 1687, 1621, $1588,1521,1506,1488,1288,1338 \mathrm{~cm}^{-1}$; ${ }^{1} \mathrm{H} \mathrm{NMR}\left(\mathrm{CDCl}_{3}, 600 \mathrm{MHz}\right) \delta: 2.38\left(\mathrm{~s}, 3 \mathrm{H}, \mathrm{CH}_{3}\right), 3.79(\mathrm{~d}, J=15.98$ $\left.\mathrm{Hz}, 1 \mathrm{H}, \mathrm{COCH}_{2} \mathrm{~S}\right), 4.09\left(\mathrm{~m}, 2 \mathrm{H}, \mathrm{SCH}_{2}\right), 4.30\left(\mathrm{~d}, J=16.14 \mathrm{~Hz}, 1 \mathrm{H}, \mathrm{COCH}_{2} \mathrm{~S}\right), 6.34(\mathrm{dd}, J=3.21,1.82 \mathrm{~Hz}, 1 \mathrm{H}$, furan-H), 6.44 (d, $J=3.22 \mathrm{~Hz}, 1 \mathrm{H}$, furan-H), 6.81 (s, $1 \mathrm{H}, \mathrm{NCHS}), 7.00$ (dd, $J=7.88,1.29 \mathrm{~Hz}, 1 \mathrm{H}, \mathrm{Ar}-\mathrm{H}), 7.20$ $(\mathrm{d}, J=7.89 \mathrm{~Hz}, 1 \mathrm{H}$, Ar-H), $7.38(\mathrm{~d}, J=1.01 \mathrm{~Hz}, 1 \mathrm{H}$, furan-H), $7.65(\mathrm{~s}, 1 \mathrm{H}$, Ar-H); MS (APCI) $m / z: 386.8$ $(\mathrm{M}+\mathrm{H})^{+}$. Anal. Calcd for $\mathrm{C}_{18} \mathrm{H}_{14} \mathrm{~N}_{2} \mathrm{O}_{2} \mathrm{~S}_{3}$ : C 55.93, H 3.65, N 7.25, S 24.89; Found C 55.89, H 3.60, N 7.20, S 24.80 .

\subsubsection{3-(8-Fluoro-4H-thiochromeno[4,3-d]thiazol-2-yl)-2-(furan-2-yl)thiazolidin-4-one (3i)}

Yield $64 \%$ of brown solid, m.p. $176-179{ }^{\circ} \mathrm{C}$, UV-vis $(\mathrm{MeOH}) \lambda_{\max }: 234 \mathrm{~nm}$; IR (KBr) $v: 1683,1615,1521$, 1506, $1288 \mathrm{~cm}^{-1}$; ${ }^{1} \mathrm{H} \mathrm{NMR}\left(\mathrm{CDCl}_{3}, 600 \mathrm{MHz}\right) \delta: 3.77$ (d, $\left.J=16.06 \mathrm{~Hz}, 1 \mathrm{H}, \mathrm{COCH}_{2} \mathrm{~S}\right), 4.14-4.04(\mathrm{~m}, 2 \mathrm{H}), 4.28$ $\left(\mathrm{d}, J=16.13 \mathrm{~Hz}, 1 \mathrm{H}, \mathrm{COCH}_{2} \mathrm{~S}\right), 6.33(\mathrm{dd}, J=3.28,1.84 \mathrm{~Hz}, 1 \mathrm{H}$, furan-H), $6.43(\mathrm{~d}, J=3.25 \mathrm{~Hz}, 1 \mathrm{H}$, furan- $\mathrm{H})$, 6.74 (s, 1H, NCHS), 7.11 (dd, $J=8.32,2.39 \mathrm{~Hz}, 1 \mathrm{H}, \mathrm{Ar}-\mathrm{H}), 7.20$ (d, $J=8.31 \mathrm{~Hz}, 1 \mathrm{H}, \mathrm{Ar}-\mathrm{H}), 7.36$ (d, $J=1.08$ $\mathrm{Hz}, 1 \mathrm{H}$, furan-H), $7.76(\mathrm{~d}, J=2.32 \mathrm{~Hz}, 1 \mathrm{H}, \mathrm{Ar}-\mathrm{H})$; MS (APCI) $m / z: 390.8(\mathrm{M}+\mathrm{H})^{+}$. Anal. Calcd for $\mathrm{C}_{17} \mathrm{H}_{11} \mathrm{FN}_{2} \mathrm{O}_{2} \mathrm{~S}_{3}: \mathrm{C}$ 52.29, H 2.84, N 7.17, S 24.64; Found C 52.23, H 2.90, N 7.22, S 24.60.

2.2.10 3-(7-Fluoro-8-methyl-4H-thiochromeno[4,3-d]thiazol-2-yl)-2-(furan-2-yl)thiazolidin-4-one (3j) Yield 61\% of brown solid, m.p. $169-172{ }^{\circ} \mathrm{C}$, UV-vis (MeOH) $\lambda_{\max }: 238 \mathrm{~nm}$; IR (KBr) $v: 2923,2852,1683,1622,1588,1506$, 1466, 1388, $1280 \mathrm{~cm}^{-1} ;{ }^{1} \mathrm{H} \mathrm{NMR}\left(\mathrm{CDCl}_{3}, 600 \mathrm{MHz}\right) \delta: 2.27\left(\mathrm{~s}, 3 \mathrm{H}, \mathrm{CH}_{3}\right), 3.79\left(\mathrm{~d}, J=16.03 \mathrm{~Hz}, 1 \mathrm{H}, \mathrm{COCH}_{2} \mathrm{~S}\right)$, 4.15-4.05 (m, 2H, $\left.\mathrm{SCH}_{2}\right), 4.31\left(\mathrm{~d}, J=15.98 \mathrm{~Hz}, 1 \mathrm{H}, \mathrm{COCH}_{2} \mathrm{~S}\right), 6.34(\mathrm{dd}, J=3.07,1.87 \mathrm{~Hz}, 1 \mathrm{H}$, furan-H), 6.45 $(\mathrm{d}, J=3.24 \mathrm{~Hz}, 1 \mathrm{H}$, furan-H), $6.76(\mathrm{~s}, 1 \mathrm{H}, \mathrm{NCHS}), 7.12(\mathrm{~d}, J=7.22 \mathrm{~Hz}, 1 \mathrm{H}$, furan-H), $7.38(\mathrm{~s}, 1 \mathrm{H}, \mathrm{Ar}-\mathrm{H}), 7.46$ (d, $J=10.26 \mathrm{~Hz}, 1 \mathrm{H}, \mathrm{Ar}-\mathrm{H})$; MS (APCI) $m / z: 404.8(\mathrm{M}+\mathrm{H})^{+}$. Anal. Calcd for $\mathrm{C}_{18} \mathrm{H}_{13} \mathrm{FN}_{2} \mathrm{O}_{2} \mathrm{~S}_{3}: \mathrm{C}$ 53.45, H 3.24, N 6.93, S 23.78; Found C 53.40, H 3.20, N 6.97, S 23.74.

\subsection{Preparation of compounds 4}

Compound $3(1.00 \mathrm{mmol})$ was dissolved in the THF $(10 \mathrm{~mL})$ and cooled to $0{ }^{\circ} \mathrm{C}$, then added $\mathrm{NaH}(2.50 \mathrm{mmol})$. After stirring about $10 \mathrm{~min}$, aromatic aldehyde $(1.10 \mathrm{mmol})$ added to the solution. Keeping the reaction at room temperature over $2 \mathrm{~h}$, then the mixture was cooled to $0{ }^{\circ} \mathrm{C}$ and poured into water $(4 \mathrm{~mL})$. After the solution was extracted with ethyl acetate, the organic layer was dried over anhydrous $\mathrm{MgSO}_{4}$. Solvent was removed under reduced presser to provide a crude product which was purified by column chromatography (silica-gel, 
dichloromethane: petrol- eum=1:8-1:1(v/v)) to afford pure 4 .

2.3.1 3-(8-Chloro-4H-thiochromeno[4,3-d]thiazol-2-yl)-2-(furan-2-yl)-5-(furan-2-ylmethylene)thiazol-

idin-4-one (4)

Yield $80 \%$ of green solid, m.p. $220-223{ }^{\circ} \mathrm{C}$, UV-vis (MeOH) $\lambda_{\max }: 245 \mathrm{~nm}$; IR (KBr) $v: 1685,1621,1588,1506$, $1466,1280 \mathrm{~cm}^{-1}$; ${ }^{1} \mathrm{H}$ NMR $\left(\mathrm{CDCl}_{3}, 600 \mathrm{MHz}\right) \delta: 4.08\left(\mathrm{~d}, J=15.04 \mathrm{~Hz}, 1 \mathrm{H}, \mathrm{SCH}_{2}\right), 4.14(\mathrm{~d}, J=15.03 \mathrm{~Hz}, 1 \mathrm{H}$, $\left.\mathrm{SCH}_{2}\right), 6.33$ (dd, $J=9.57,3.17 \mathrm{~Hz}, 1 \mathrm{H}$, furan-H), 6.55-6.49 (m, $2 \mathrm{H}$, furan-H), $6.66(\mathrm{~d}, J=3.44 \mathrm{~Hz}, 1 \mathrm{H}$, furan-H), 6.88 (s,1H, NCHS), 7.09 (dd, $J=8.29,2.26 \mathrm{~Hz}, 1 \mathrm{H}, \mathrm{Ar}-\mathrm{H}), 7.18(\mathrm{~d}, J=8.28 \mathrm{~Hz}, 1 \mathrm{H}, \mathrm{Ar}-\mathrm{H}), 7.34(\mathrm{~s}$, $1 \mathrm{H}, \mathrm{Ar}-\mathrm{H}), 7.54(\mathrm{~d}, J=8.61 \mathrm{~Hz}, 1 \mathrm{H}$, furan-H), $7.59(\mathrm{~s}, 1 \mathrm{H}, \mathrm{Ar}-\mathrm{H}), 7.72(\mathrm{~d}, J=2.24 \mathrm{~Hz}, 1 \mathrm{H}$, furan-H); MS (APCI) $m / z$ : $484.7(\mathrm{M}+\mathrm{H})^{+}, 486.7(\mathrm{M}+2+\mathrm{H})^{+}$. Anal. Calcd for $\mathrm{C}_{22} \mathrm{H}_{13} \mathrm{ClN}_{2} \mathrm{O}_{3} \mathrm{~S}_{3}$ : C 54.48, H 2.70, N 5.78, S 19.83; Found C 54.43, H 2.67, N 5.82, S 19.79.

\subsection{Anticancer Activity in Vitro}

The anticancer activities of target compounds were evaluated in vitro gastric cancer cells (SGC-7901), cervical cancer cells (Hela) and lung cancer cells (A-549) by measuring cell viability according to the MTT method described in the literature with cisplatin as the positive control. The cells were seeded in RPMI 1640 medium $(100 \mu \mathrm{L})$ in a 96-well plate at a concentration of $6000-7000$ cells per well. After culturing for $12 \mathrm{~h}$ at $37{ }^{\circ} \mathrm{C}$ and $5 \%$ $\mathrm{CO}_{2}$, cells were incubated with various concentrations of the samples for $24 \mathrm{~h}$. Twenty microliter of MTT ( 5 $\mathrm{mg} / \mathrm{mL}$ ) was added and incubated with the cells for $4 \mathrm{~h}$. The formazan product was dissolved by adding dimethyl sulfoxide (DMSO, $100 \mu \mathrm{L}$ ) to each well, and the plates were read at $570 \mathrm{~nm}$. All measurements were performed in triplicate and each experiment was repeated at least three times. $\mathrm{IC}_{50}$ values were determined as the drug and sample concentration at $50 \%$ inhibition of the cell growth.

\section{Results and discussion}

\subsection{Microwave-assisted synthesis thiazolidin-4-ones}

Generally, 1,3-thiazolidin-4-ones derivatives can be synthesized by a three-component condensation of a primary amine, an aldehyde, and mercaptoacetic acid. The dehydration process is the rate determining step of the reaction which is usually performed, the reaction is usually performed in refluxing toluene with dehydrating agents for 2-48 hours, but the yields were generally not satisfactory, Thus either a catalyst or a dehydrating agent or a Dean and Stark' was usually used to promote reaction. The applications of some new synthetic methods, such as microwave-assisted synthesis, and reagents, such as ionic liquids (Zhang, Xinying, 2009), and KSF clay (Dandia, A., 2006), have been recently explored for improving the synthesis of the 1,3-thiazolidin-4-ones. We now reported a new method for one-pot synthesis of a series of new 1,3-thiazolidin-4-ones using DCC as a dehydrating agent under microwave heating in a sealed vessel (Scheme 1).

This new method resulted in increased yields with shorter reaction times in comparison with the conventional routes which typically affords yields in the range $10 \%-65 \%$. As to different substitutions of aromatic aldhydes $\mathrm{R}_{2}-\mathrm{CHO}$, their reactivity was: $\mathrm{R}_{2}=$ aryl with electron withdrawing $>$ aryl with no substitution $>$ aryl with electron donating. The yields were good when aromatic aldhydes with electron withdrawing, such as $-\mathrm{NO}_{2}$ and $-\mathrm{Cl}$, and it was more than $85 \%$; when $\mathrm{R}_{2}=4$-nitrophenyl, the yield was the best. When aromatic aldhydes with electron donating, mainly because electron donating groups increased the electron density reducing the reactivity of carbonyl, so the reactivity lower, the yield decreased. In this report, we also studied the effects of different substituents on $4 H$-thiochromeno[4,3- $d$ ] thiazol-2-amines. The results showed that different $\mathrm{R}$ and $\mathrm{R}_{1}$ had no significant impact on the yield of 1,3-thiazolidin-4-one.

In conclusion, during the microwave-assisted synthesis of 1,3-thiazolin-4-ones, the electron density of aldhydes had great impact on the reactivity. Generally speaking, the reactivity was higher when aromatic aldhydes with electron withdrawing, and the yield was also good; when electron donating on aromatic ring, the reactivity was lower, so the yield decreased.

\subsection{Study for anticancer activity}

Anticancer activities were tested against gastric cancer cells (SGC-7901), cervical cancer cells (Hela) and lung cancer cells (A-549) and using cisplatin as positive control (Table 1). Most of the compounds had good anticancer activities. $R_{2}$ had significant impact on the anticancer activity. The $R_{2}$ with deactivating groups, such as $R_{2}=4$-nitrophenyl and 4-chlorophenyl (compounds $3 \mathrm{c}$ and $3 \mathrm{f}$ ) had better anticancer activiy than that had activating groups or no substitutions. When $R_{2}$ was maintained, different $R$ and $R_{1}$ had no significant impact on the anticancer activity. Moreover it was worth noting that the anticancer activity was better when $\mathrm{R}_{2}$ was heterocyclic than $\mathrm{R}_{2}$ was benzene ring's. In addition, the further molecular modifications on 
1,3-thiazolidin-4-one brought us better result and methylene at C-5 position of 1,3-thiazolidin-4-one showed the best anticancer activity.

In summary, a series of new 1,3-thiazolidin-4-ones synthesized under microwave-assisted with DCC as dehydrating agent were reported. In microwave-assisted synthesis conditions, the yields of the new compounds were better than conventional method and the reaction time in 10 minutes. Most of the new compounds had anticancer activity, compound had the best anticancer activity when a furan-2-yl was at C-2 position and a methylene was at C-5 position, so it may be noted that different heteroaryl substituents at C-2 position and methylene at C-5 position of 1,3-thiazolidin-4-ones deserve further consideration from the point of structure-activity optimization. Further structure-activity optimization and other biological studies of 1,3-thiazolidin-4-ones are undertook in the laboratory.

\section{References}

Aridoss, G., Amirthaganesan, S., Kim, M. S., Kim, J. T., \& Jeong, Y. T. (2009). Synthesis, spectral and biological evaluation of some new thiazolidinones and thiazoles based on t-3-alkyl-r-2,c-6-diarypip- eridin-4-ones. European Journal of Medicinal Chemistry, 44, 4199-4210.

Balzarini, J., Orzeszko, B., Maurin, J. K., \& Orzeszko, A. (2007). Synthesis and anti-HIV studies of 2-adamantyl-substituted thiazolidin-4-ones. European Journal of Medicinal Chemistry, 42, 993-1003.

Chen, H., Jiao, L. L., Guo, Z. H., Li, X. L., Ba, C. L., \& Zhang, J. C. (2008). Synthesis and biological activity of novel thiazolidin-4-ones with a carbohydrate moiety. Carbohydrate Research, 343, 3015-3020.

Dandia, A., Singh, R., Khaturia, S., Merienne, C., Morgant, G., \& Loupy, A. (2006). Efficient microwave enhanced regioselective synthesis of a series of benzimidazolyl/triazolyl spiro- [indole-thiazolidinones] as potent antifungal agents and crystal structure of spiro[3Hindole-3,2'-thiazolidine]-3'(1,2,4-triazol-3-yl)-2,4'(1H)-dione. Bioorganic \& Medicinal Chemistry, 14, 2409-2417.

Küçükgüzel, G., Kocatepe, A., Clercq, E. D., Şahin, F., \& Güllüce, M. (2006). Synthesis and biological activity of 4-thiazolidinones, thiosemicarbazides derived from diflunisal hydrazide. European Journal of Medicinal Chemistry, 41, 353-359.

Mujeebur, R.V. P., Mukhtar, S., Ansari, W. H., \& Lemiere, G. (2005). Synthesis, stereochemistry and biological activity of some novel longalkyl chain substituted thiazolidin-4-ones and thiazan-4-one from-undecenoic acid hydrazide. European Journal of Medicinal Chemistry, 40, 173-184.

Murugesan, V., Prabhakar Y. S., \& Katti, S. B. (2009). CoMFA and CoMSIA studies on thiazolidin-4-one as anti-HIV-1 agents. Journal of Molecular Graphics and Modelling, 27, 735-743.

Ottana, R., Carotti, Maccari, S., Landini, R. I., Chiricosta, G., Caciagli, B., Vigoritaa, M.G., \& Mini, E. (2005). In vitro antiproliferative activity against human colon cancer cell lines of representative 4-thiazolidinones. Bioorganic \& Medicinal Chemistry, 15, 3930-3933.

Ottana, R., Maccari, R., Barreca, M. L., Bruno, G., Rotondo, A., Rossi, A., Giuseppa, C., Paola, R. D., Sautebin, L., Cuzzocrea, S., \& Vigorita, M. G. (2005). 5-Arylidene-2-imino-4-thiazolidinones: Design and synthesis of novel anti-inflammatory agents. Bioorganic \& Medicinal Chemistry, 13, 4243-4252.

Ravindra, K. R., Rajkamal, T., Katti, S. B., Christophe, P., \& Erik D C. (2007). Design, synthesis, and evaluation of 2-aryl-3-heteroaryl-1,3-thiazolidin-4-ones as Anti-HIV Agents. Bioorganic \& Medicinal Chemistry, 15, 1725-1731.

Ravindra, K. R., Rajkamal, T., Katti, S. B., Christophe, P., \& Erik, D. C. (2007). Design, synthesis, and evaluation of 2-aryl-3-heteroaryl-1,3-thiazolidin-4-ones as anti-HIV agents. Bioorganic \& Medicinal Chemistry, $15,1725-1731$.

Vicini, P., Geronikaki, A., Anastasia, K., Incerti, M., \& Zani, F. (2006). Synthesis and antimicrobial activity of novel 2-thiazolylimino-5-arylidene-4-thiazolidinones. Bioorganic \& Medicinal Chemistry, 14, 3859-3864.

Vicini, P., Geronikaki, A., Incerti, M., Zani, F., Dearden, J., \& Hewitt, M. (2008). 2-Heteroarylimino5-benzylidene-4-thiazo-lidinones nalogues of 2-thiazolylimino-5-benzylidene-4-thiazolidinones with antimicrobial activity: Synthesis and structure-activity relationship. Bioorganic \& Medicinal Chemistry, 16, 3714-3724.

Zhan, F. X., Zhao, G. L., Wang, Y. L., Xu, W. R., Tang, L. D., \& Wang, J. W. (2009). Design, synthesis and anti-diabetic activities of thiazole-bearing compounds as novel dipeptidyl peptidase IV inhibitors. Chinese 
Journal of Medical Chemistry, 19, 334-339.

Zhang, X. Y., Li, X. Y., Li, D. F., Qu, G., Wang, J. J., Loiseau, P. M., \& Fan, X. S. (2009). Ionic liquid mediated and promoted eco-friendly preparation of thiazolidinoneand pyrimidine nucleoside-thiazolidinone hybrids and their antiparasitic activities. Bioorganic \& Medicinal Chemistry Letter, 19, 6280-6287.

Table 1. Anticancer activity of compounds synthesized

\begin{tabular}{cccc}
\hline Compounds & \multicolumn{3}{c}{$\mathrm{IC}_{50}(\mu \mathrm{M})$} \\
\cline { 2 - 4 } & $\mathrm{SGC}-7901$ & Hela & $\mathrm{A}-549$ \\
\hline $3 \mathrm{a}$ & 17.60 & 19.00 & 19.60 \\
$3 \mathrm{~b}$ & 8.35 & 8.85 & 9.58 \\
$3 \mathrm{c}$ & 12.78 & 14.08 & 13.43 \\
$3 \mathrm{~d}$ & 17.00 & 16.55 & 18.95 \\
$3 \mathrm{e}$ & 16.00 & 17.66 & 18.79 \\
$3 \mathrm{f}$ & 10.42 & 10.20 & 10.92 \\
$3 \mathrm{~g}$ & 19.34 & 20.39 & 19.31 \\
$3 \mathrm{~h}$ & 9.08 & 9.00 & 10.01 \\
$3 \mathrm{i}$ & 9.89. & 8.90 & 9.79 \\
$3 \mathrm{j}$ & 9.97 & 9.10 & 10.04 \\
4 & 5.76 & 6.19 & 6.67 \\
Cisplatin & 5.05 & 4.66 & 7.70 \\
\hline
\end{tabular}

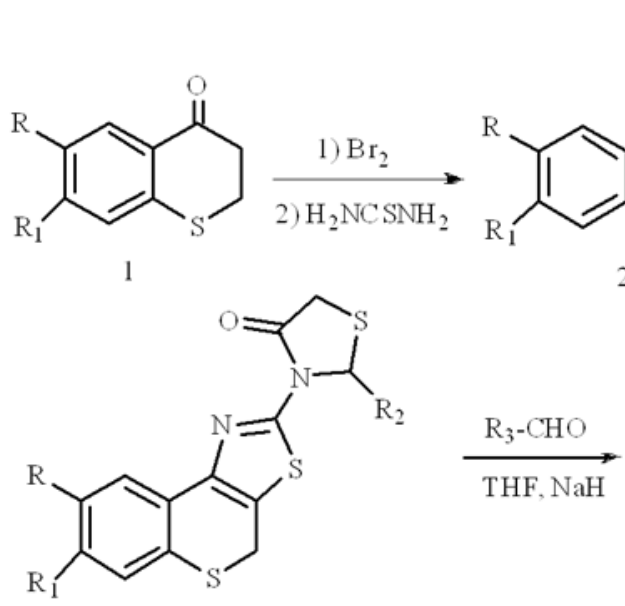

3<smiles>[R3]C=C1SC([R])N(c2nc3c(s2)CSc2cc([R])c([R])cc2-3)C1=O</smiles>

4

1a: $\mathrm{R}=\mathrm{Cl}, \mathrm{R}_{1}=\mathrm{H} ; \quad$ b: $\mathrm{R}=\mathrm{CH}_{3}, \mathrm{R}_{1}=\mathrm{H} ; \quad 1 \mathrm{c}: \mathrm{R}=\mathrm{F}, \mathrm{R}_{1}=\mathrm{H} ; \quad 1 \mathrm{~d}: \mathrm{R}=\mathrm{CH}_{3}, \mathrm{R}_{1}=\mathrm{F}$

2a: $\mathrm{R}=\mathrm{Cl}, \mathrm{R}_{1}=\mathrm{H} ; \quad 2 \mathrm{~b}: \mathrm{R}=\mathrm{CH}_{3}, \mathrm{R}_{1}=\mathrm{H} ; \quad 2 \mathrm{c}: \mathrm{R}=\mathrm{F}, \mathrm{R}_{1}=\mathrm{H} ; \quad 2 \mathrm{~d}: \mathrm{R}=\mathrm{CH}_{3}, \mathrm{R}_{1}=\mathrm{F}$

3a: $\mathrm{R}=\mathrm{Cl}, \mathrm{R}_{1}=\mathrm{H}, \mathrm{R}_{2}=4$-methoxyphenyl; $3 b: \mathrm{R}=\mathrm{Cl}, \mathrm{R}_{1}=\mathrm{H}, \mathrm{R}=$ = furan-2-yl; $3 \mathrm{c}: \mathrm{R}=\mathrm{Cl}, \mathrm{R}_{1}=\mathrm{H}, \mathrm{R}_{2}=4$-nitrophenyl;

3d: $\mathrm{R}=\mathrm{Cl}, \mathrm{R}_{1}=\mathrm{H}, \mathrm{R}_{2}=$ phenyl; 3e: $\mathrm{R}=\mathrm{Cl}, \mathrm{R}_{1}=\mathrm{H}, \mathrm{R}_{2}=4$-(dimethy la mino) phenyl; $3 \mathrm{f}: \mathrm{R}=\mathrm{Cl}, \mathrm{R}_{1}=\mathrm{H}, \mathrm{R}_{2}=4$-chlorophenyl;

$3 \mathrm{~g} . \mathrm{R}=\mathrm{Cl}, \mathrm{R}_{1}=\mathrm{H}, \mathrm{R}_{2}=$ benzo[d] [1,3]dioxole-5-yl; 3h: $\mathrm{R}=\mathrm{CH}_{3}, \mathrm{R}_{1}=\mathrm{H}, \mathrm{R}_{2}=$ furan-2-yl; 3i: $\mathrm{R}=\mathrm{F}, \mathrm{R}_{1}=\mathrm{H}, \mathrm{R}_{2}=$ furan-2-yl;

3j: $\mathrm{R}=\mathrm{CH}_{3} \cdot \mathrm{R}_{1}=\mathrm{F}, \mathrm{R}_{2}=$ furan -2 - $\mathrm{yl}$

4: $\mathrm{R}=\mathrm{Cl}, \mathrm{R}_{1}=\mathrm{H}, \mathrm{R}_{2}=$ furan $-2-\mathrm{yl}, \mathrm{R}_{3}=$ furan-2-yl

Scheme 1. The synthesis route of target compound 